
\title{
28 Research Square \\ Comparison of single-layered continuous and interrupted gastrointestinal anastomosis in pediatric patients
}

Qianyang Liu

Chongqing Medical University Affiliated Children's Hospital

Ping Li

Qingdao University Medical College

Chunbao Guo ( $\nabla$ guochunbao@foxmail.com )

Chongqing Medical University

Research article

Keywords: Single-layered continuous anastomosis; Interrupted anastomosis; Anastomotic leakage;

Postoperative complications; Overall expenditure

Posted Date: September 11th, 2019

DOI: https://doi.org/10.21203/rs.2.14304/v1

License: (c) (i) This work is licensed under a Creative Commons Attribution 4.0 International License.

Read Full License 


\section{Abstract}

Background Optimal gastrointestinal construction in pediatric patients still exist controversy as to the technique for the anastomosis. The study was to investigate the advantage of single-layered continuous anastomosis for pediatric patients who underwent intestinal anastomosis on emergency or electively laparotomy.

Methods The medical records of 252 patients with single-layered continuous anastomosis and 196 patients with two-layered interrupted anastomosis, served as control, were reviewed retrospectively. Clinical outcomes, including postoperative complications, overall expenditure, postoperative hospital stay and anastomotic leakage, were compared according to the single-layered or two-layered anastomosis.

Results Single-layered continuous anastomosis was associated with advantageous postoperative outcomes, including anastomotic time $(p<0.001)$, operative time $(p=0.041)$ and overall expenditure $(p=0.009)$. A reduction trend for postoperative hospital stay was indicated in patients receiving singlelayer anastomoses $(p=0.09)$. There was also an decreased of inflammation variables, C-reactive protein [CRP]) at postoperative days (POD) $5(p=0.032)$ in the single-layer anastomoses patients. There were no differences for the other main complications.

Conclusions Beneficial effects of single-layered continuous anastomosis in terms of anastomotic time, faster postoperative recovery and cost incurred in pediatric patients were demonstrated.

\section{Background}

The methods of reconstruction and anastomosis for digestive tract influence the postoperative outcome and complications, including leakage, intestinal fistula and stricture $[1,2,3,4,5]$. The traditional manual anastomosis method is a complex surgery, consisting of double-layer interrupted silk suture and a running absorbable suture, which requires an experienced surgeon ${ }^{[6,7]}$. Furthermore, the construction using double-layer interrupted silk suture is mentioned tedious and time-consuming to perform ${ }^{[8]}$.

The single-layer continuous anastomosis have advocated in several reports, with less time to construction, less economic cost ${ }^{[9,10]}$. It has been confirmed that this methods could as safe as, even superior to conventional double-layer suturing for its advantage of simplicity ${ }^{[11]}$. However, the efficacy of the method combined with continuous suture in pediatric patients remains unknown.

In our institute, single-layer continuous suture method was piloted used in some patients by some surgeon on duty. Therefore, we retrospectively investigated the efficacy of the single-layer continuous anastomosis method for pediatric patients compared with traditional manual anastomosis.

\section{Methods}

Patients 
This study is a retrospective review of the medical records of a series of patients that underwent intestinal anastomosis on emergency or electively laparotomy in our institutions from 2010 to Aug. 2018. The study was approved by the chongqing children's Institutional Review Board in using the medical records. The patients were considered eligible for entry into the study with primary intestinal anastomosis. All the patients should be aged between 1 year and 14 years. Exclusion criteria included patients requiring anastomosis involving the stomach, duodenum, or the rectum, and those with enteroplasty or proximal stom. Additionally, to minimize severity differences in the study population, patients managed in the intensive care unit (ICU) for more than 3 day were excluded. Of the 448 patients who met the inclusion criteria, the clinical and pathological details of all the cases were carefully recorded. Baseline data collected included age, gender, diagnosis, and $\mathrm{Hb}$ level prior to the operations. Treatment-related data included type of operation, operating time, estimated blood loss (EBL), transfusion rate and necessity for re-operation were also recorded. The surgical time was recorded from the first skin incision to the last suture placement for all the techniques.

\section{Gastrointestinal anastomosis technique}

The recruited patients were received anastomosis by two parallel, synchronous sewing methods: several groups using the interrupted sutureanastomosis in comparison to the continuous suture. All surgeries were performed under general anaesthesia by the consultant surgeons in our institute. The diseased bowel segment was removed using the standard technique, end-to-end or end to side construction were performed. The single-layered intestinal anastomoses were performed using a continuous 5-0 absorbable PDS suture (Ethicon Inc., Norderstedt, German) that began at the mesenteric border. The edge distance and needle pitch were approximately $3-5 \mathrm{~mm}$ of each bite, with all the layers incorporating. Only appropriate pressure could be subjected to the suture to avoid ischemia of the anastomosis while render the anastomosis water tight. The edges of the mesentery were conventionally closed. The double-layer interrupted suture was constructed using 5-0 silk suture for the inner transmural layer. The outer seromuscular were also taken in a interrupted manner.

\section{Postoperative management and complications}

All patients underwent the same postoperative programme, including fluid resuscitation, parenteral nutrition support, perioperative intravenous broad-spectrum antibiotics (average 4.32 days), including semisynthetic penicillins, cephalosporins, carbapenems, and metronidazole, enteral feeding, early ambulation and intensive care therapy (cardiorespiratory support or fluid resuscitation) if necessary. Total parenteral nutrition, intravenous fluids ( $5 \%$ dextrose $/ 0.5 \%$ normal saline solution), $\mathrm{H}_{2}$ blockers and other electrolytes were prescribed as indicated clinically.

The primary outcome measure was the incidence of anastomotic leak, and the overall postoperative outcomes. Secondary outcomes included overal expenditure, and length of hospital stay, in days. We described the surgical and postoperative outcomes, including postoperative gastrointestinal function recovery, most common postoperative complications, and total lengths of hospital stay (the number of 
days from the day of operation until the date of discharge). The postoperative complications, including complication rates, complication types, such as wound infection, intra-abdominal or pelvic abscesses, anastomotic leaks, and the number of re-operation were also recorded. Early ileus was defined as more than 1 episode of nausea or vomiting within the hospital stay. The clinical leak was defined as the development of systemic sepsis associated with local peritoneal signs, the appearance of fecal material in the abdominal drains.

From January 2018 to December 2018, we have consecutively recorded the surgical time required for construction of the anastomosis in respective 10 patients of the two methods. The time recorded for construction of the anastomosis began with the placement of the first stitch and ended with cutting the excess material from the last stitch.

\section{Statistical methods}

We first measured the demographic and preoperative clinical data and intraoperative factors to compare the two groups, then, the surgical and postoperative outcomes were subjected to statistical comparisons using SPSS 20.0 (IBM, Armonk, NY). Continuous data were presented as medians and interquartile ranges (IQR) or means \pm SDs as appropriate and analyzed with Student's t-test or the Mann-Whitney U test, respectively. Categorical variables were reported as frequencies (percentages) and were analyzed by a chi-square test or Fisher's exact test. The statistical significance was evaluated using a two-tailed $95 \%$ confidence interval $(\mathrm{Cl})$, and statistical significance was established if $p<0.05$.

\section{Results}

\section{Patient characteristics}

At the time of the analysis, a total of 448 pediatric patients were enrolled for analysis. Among them, 252 (56.3\%) received single-layer anastomoses and 196(43.7\%) did two-layer anastomoses. The baseline features of the pediatric patients according to single-layer anastomoses or two-layer anastomoses are summarized in Table 1 . The groups were evenly matched with respect to demographic, preoperative and intraoperative factors (Table 1). In addition, there were no significant differences in location of the anastomosis between the two groups.

\section{Clinical outcomes}

Table 2 illustrates the clinical outcomes based on single-layer anastomoses and two-layer anastomoses. Following the surgery, there were no significant differences between the two groups with regard to the estimated blood loss $(E B L)(p=0.41)$, transfused patients $(p=0.38)$, and early ileus $(p=0.44)$. There was significant difference in the mean time required for single-layer anastomosis $(p<0.01)$ and the totle operative time $(p=0.041)$. 
The overall expenditure included the apparatus, thread and drugs in the single-layered group was about $23.8 \pm 9.4\left({ }^{*} 10^{3}\right)$ Chinese yuan, whereas in the double-layered group, it was $27.4 \pm 11.2\left({ }^{*} 10^{3}\right)$ Chinese yuan $(p=0.009)$ (Table 2), although for the anastomosis expenditure, it was higher in the single-layer anastomoses (100 Chinese yuan)(1 pack of 5-0 PDS suiture) compared to that in the two-layer anastomoses (21 Chinese yuan)(2 pack of 3-0 silk suture) group. For postoperative inflammation variables, C-reactive protein [CRP]), significant differences were found between the two groups at POD 5 $(p=0.032)$.

\section{Postoperative complications}

According to established criteria, postoperative complications are summarized in detail in Table 3 . There were no statistically significant differences between the two groups for the total complications episodes $(p=0.34)$, postoperative anastomotic leakage $(p=0.38)$, wound infection $(p=0.41)$, sepsis $(p=0.37)$, and peritonitis or abscess $(p=0.46)$, which should be subjected to surgical intervention.

\section{Discussion}

The present study assessed the efficacy and safety of the continuous single-layer anastomosis suture compared with the interrupted two-layer anastomoses after the gastrointestinal resection, mainly in terms of anastomotic leak, time required to construct the anastomosis, cost incurred, and length of hospital stay. We used the single-layer continuous suture in the anastomosis of the digestive tract in 448 cases and demonstrated that single-layer suture appeared to be more efficient than the traditional two-layer techniques. The complication rate or anastomotic leak incidence of single-layer anastomosis were the same or lower compared to that observed with traditional interrupted two-layer anastomotic techniques ${ }^{[11,12]}$. Moreover, the overall expenditure was evidently reduced.

For gastrointestinal anastomosis, the suitability is to heal without leakage, which are under the direct control of the surgeon ${ }^{[13]}$. Leakage rates tended to be lower with more experienced surgeons, and poor technique are clearly responsible for anastomotic failure ${ }^{[14,15,16]}$. Undoubtedly, the continuous suture is the tightest, which is commonly used for blood vessel sutures. Furthermore, single-layer anastomosis is as strong as double-layer suturing to ensure mechanical integrity in the small intestine and colon anastomosis ${ }^{[17,18]}$. In the current research, the anastomotic leak and abdominal abscess rate in the current research were comparable for the continuous single-layer suture compared with the control and to the literature available ${ }^{[18]}$. In the double-layered technique, there may be narrowing of the lumen and stricture formation due to excessive tissue inversion ${ }^{[22]}$, which could be avoided in the single-layered technique, as only one layer of sutures is incorporated. Due the extremely low incidence of anastomotic strictures, we really did not recorded any case for either of this two methods of anastomotic comstruction. In addition, the continuous suture was proven to contribute to adjustment of the

anastomostic diameter, which may expand and contract according to the intraluminal forces ${ }^{[23]}$. All this may explain the rarity of anastomotic leak of the suture line. 
In our institute, a absorbable monofilament suture line was used which is related to the properties of less tissue reaction in a continuous fashion. Also, the edges of intestinal were pruned with an adequate blood supply for the continuous anastomosis ${ }^{[19,20]}$. Of all the anastomoses studied, the blood flow of the suture line decreased from the single-layered manual to the two-layered manual to the stapled suture. Although the inner layer of double-layered technique might be hemostatic, it may cause the mucosa strangulation. This is avoided in single-layered technique, as suture is taken sparing mucosa, and hence causing lower damage to the submucosal vascular plexus ${ }^{[21]}$. Although it is unknown whether the observed effects can be attributed to the single-layered metnod, the slightly decrease for the CRP at POD 5 in the single layered group might reflect the faster recovery from the inflammatory infiltrate. In an experimental setting, it was in favor of single-layered method for a decreasing number of inflammation and fibroblast cells compared with the two layers of anastomosis suture groups, which might be involved with fibrosis and gastrointestinal function ${ }^{[24]}$.

Due to emergency basis of the current procedures, it seemed beneficial to the patients to reduce construction time, the time of anesthesia ${ }^{[26]}$. Single-layer continuous anastomosis is an easy technique without a painful learning curve. The mean time documented in constructing the single-layered anastomosis was greatly shortened, which should contribute to the overall operation time. Other report also indicated the same difference for the timings of the anastomosis using the single-layer continuous method ${ }^{[25,2,16]}$. Although the cost of materials for the single-layer anastomoses was higher than twolayered anastomosis, it was surprising to see the total cost of the single-layer anastomoses was significant lower, which might come from the shorter procedure time and hospital stay by the rapid recovery from the single-layer anastomoses with the population here in our set-up, which was definitely significant.

There are several weaknesses to our study. First, the results of this study were not made randomly. Furthermore, this is a single-center study, in which we collected the data with inherent risk of selection bias. The study also takes place over a long time period and outcomes from many patients may not reflect outcomes from current treatment algorithms, there have likely been many practice changes within both the surgery and the ICU divisions, leading to different care practices between study patients. Practitioners are likely to initiate single-layer anastomoses in severe patients. These unmeasured variables may have affected our results as residual confounders. Therefore, our results need to be carefully interpreted.

\section{Conclusions}

In conclusion, the current study suggested that the single-layer continuous anastomosis did not carry any increased risk of complications, required less time for construction and less overall expenditurea s compared with its conventional counterpart.

\section{List Of Abbreviations}




\section{$\mathrm{Cl}=$ confidence interval, $\mathrm{RR}=$ risk ratio, $\mathrm{OR}=$ odd ratio, $\mathrm{CRP}=\mathrm{C}$-reactive protein, $\mathrm{POD}=$ postoperative days, ICU = intensive care unit.}

\section{Declarations}

-Ethics approval and consent to participate

The written informed consent to participate was obtained from the parents/guardians of the minors included in this study. The Institutional Review Board approval from Chongqing Children's Hospital was obtained at Aug, 25, 2017.

-Consent for publication

Not applicable-Availability of data and material

The patient's data generated or analyzed during the current study are available from the corresponding author on reasonable request. When clinical data are shared, they will be anonymized.-Competing interests

The authors declare that they have no competing interests.

-Funding

The study was funded by departmental resources.

-Authors' contributions

JL designed, analyzed the data, and evaluate the manuscript. LW performed the statistic measurement and analyzed the data. CG analyzed the data, and wrote the paper.

-Acknowledgements

We thank Prof. Xianqing Jin for providing technical assistance and for insightful discussions during the preparation of the manuscript. We thank Dr Xiaoyong Zhang at the Wistar Institute, USA, for help with the linguistic revision of the manuscript.

\section{References}

1. Slieker JC, Daams F, Mulder IM, et al. Systematic review of the technique of colorectal anastomosis. JAMA Surg. 2013; 148:190-201 
2. Law WL, Bailey HR, Max E, et al. Single-layer continuous colon and rectal anastomosis using monofilament absorbable suture (Maxon): study of 500 cases. Dis Colon Rectum. 1999; 42:736-740

3. Cikot M, Kones O, Gedikbası A, et al. The marker C-reactive protein is helpful in monitoring the integrity of anastomosis: plasma calprotectin. Am J Surg. 2016;212:53-61.

4. Masud D, Undre S, Darzi A. Using manual dexterity to predict the quality of the final product in the small bowel anastomosis after a period of training. Am J Surg. 2012;203:776-781

5. Louridas M, Szasz P, de Montbrun S, et al. Can We Predict Technical Aptitude?: A Systematic Review. Ann Surg. 2016; 263:673-691.

6. Datta V, Bann S, Mandalia M, et al. The surgical efficiency score: a feasible, reliable, and valid method of skills assessment. Am J Surg. 2006;192:372-378.

7. Moriura S, Kobayashi I, Ishiguro S, et al. Continuous mattress suture for all hand-sewn anastomoses of the gastrointestinal tract. Am J Surg. 2002;184:446-448.

8. Maan ZN, Maan IN, Darzi AW, et al. Systematic review of predictors of surgical performance. Br J Surg. 2012; 99:1610-1621.

9. Wilasrusmee C, Phromsopha N, Lertsitichai P, et al. A new vascular anastomosis model: relation between outcome and experience. Eur J Vasc Endovasc Surg. 2007;33:208-213.

10. Cossu ML, Coppola M, Fais E, et al. The use of the Valtrac ring in the upper and lower gastrointestinal tract, for single, double, and triple anastomoses: a report of 50 cases. Am Surg. 2000;66:759-762.

11. Burch JM, Franciose RJ, Moore EE, et al. Single-layer continuous versus two-layer interrupted intestinal anastomosis: a prospective randomized trial. Ann Surg. 2000;231:832-837.

12. Ordorica-Flores RM, Bracho-Blanchet E, Nieto-Zermeño J, et al. Intestinal anastomosis in children: a comparative study between two different techniques. J Pediatr Surg. 1998;33:1757-1759.

13. Oláh A, Belágyi T, Neuberger G, et al. Use of different absorbable sutures for continuous single-layer anastomosis in the gastrointestinal tract. A prospective, randomized study. Dig Surg. 2000;17:483485.

14. Kim SH, Choi HJ, Park KJ, et al. Sutureless intestinal anastomosis with the biofragmentable anastomosis ring: experience of 632 anastomoses in a single institute. Dis Colon Rectum. 2005;48:2127-2132.

15. Hussain A, Mahmood H, Nicholls J, et al. Continuous figure-of-eight suturing in upper and lower gastrointestinal anastomosis. Singapore Med J. 2008;49:672-675.

16. Max E, Sweeney WB, Bailey HR, et al. Results of 1,000 single-layer continuous polypropylene intestinal anastomoses. Am J Surg. 1991;162:461-467.

17. Sandrasegaran K, Maglinte DD, Lappas JC, et al. Small-bowel complications of major gastrointestinal tract surgery. AJR Am J Roentgenol. 2005;185:671-681.

18. Herrle F, Diener MK, Freudenberg S, et al. Single-Layer Continuous Versus Double-Layer Continuous Suture in Colonic Anastomoses-a Randomized Multicentre Trial (ANATECH Trial). J Gastrointest Surg. 2016;20:421-430. 
19. Close K, Epstein KL, Sherlock CE. A retrospective study comparing the outcome of horses undergoing small intestinal resection and anastomosis with a single layer (Lembert) or double layer (simple continuous and Cushing) technique. Vet Surg. 2014;43:471-478.

20. Orsay CP, Bass EM, Firfer B, et al. Blood flow in colon anastomotic stricture formation. Dis Colon Rectum. 1995;38:202-206.

21. Garude K, Tandel C, Rao S, et al. Single layered intestinal anastomosis: a safe and economic technique. Indian J Surg. 2013;75:290-293.

22. Park KJ, Woo JS, Jeong SS, et al. Continuous "over and over" suture for tricuspid ring annuloplasty. Korean J Thorac Cardiovasc Surg. 2012; 45:19-23.

23. Bailey HR, LaVoo JW, Max E, et al. Single-layer polypropylene colorectal anastomosis. Experience with 100 cases. Dis Colon Rectum. 1984;27:19-23.

24. Li GC, Xu Y, Zhang YC, et al. Efficacy of single-layer continuous suture of the posterior wall in anastomosis involving a difficult location of the digestive tract. Oncol Lett. 2014;8:1567-1574.

25. Khan RAA, Hameed F, Ahmed B, et al. Intestinal anastomosis: comparative evaluation for safety, cost effectiveness, morbidity and complication of single versus double layer. Professional Med J. 2010; $17: 232-234$

26. Shogan BD, Carlisle EM, Alverdy JC, et al. Do we really know why colorectal anastomoses leak? J Gastrointest Surg. 2013;17:1698-1707.

\section{Tables}

Table 1. Baseline demographics of eligible patient and preoperative variables 


\begin{tabular}{|c|c|c|c|}
\hline suture & $\begin{array}{l}\text { Continuous } \\
(252)\end{array}$ & $\begin{array}{l}\text { Interrupted } \\
(196)\end{array}$ & $\begin{array}{l}\mathrm{p} \\
\text { Values }\end{array}$ \\
\hline Age(yrs), median (interquartile ranges, IQR) & $3.8(1.2-6.8)$ & $3.7(1.1-7.1)$ & 0.34 \\
\hline Male: female & $104 \square 148$ & $86 \square 110$ & 0.32 \\
\hline \multicolumn{4}{|l|}{ Comorbidities, N (\%) } \\
\hline Shock & 28 & 20 & 0.44 \\
\hline Preoperative anemia $(\mathrm{Hb}<12 \mathrm{~g} / \mathrm{dL})$ & 89 & 65 & 0.35 \\
\hline ICU admission & 41 & 32 & 0.54 \\
\hline $\begin{array}{l}\text { Length of intestinal resection(cm), median (interquartile } \\
\text { ranges, IQR) }\end{array}$ & $12.2(3.5-31.8)$ & 11.8(3.8-29.7) & 0.28 \\
\hline \multicolumn{4}{|l|}{ Location of the anastomosis, $\mathrm{N}(\%)$} \\
\hline Small bowel resection and anastomosis & 193(76.6) & $151(77.0)$ & 0.50 \\
\hline Colon resection and right hemicolectomy & $59(23.4)$ & $45(23.0)$ & \\
\hline \multicolumn{4}{|l|}{ Operative indications, N (\%) } \\
\hline Intussusception & $98(38.9)$ & $75(38.3)$ & 0.49 \\
\hline Adhesive small bowel obstruction & $49(19.4)$ & $41(20.9)$ & 0.39 \\
\hline meckel diverticulum & $67(26.6)$ & $49(25.0)$ & 0.39 \\
\hline Incarcerated inguinal hernia & $38(15.1)$ & $31(15.8)$ & 0.47 \\
\hline
\end{tabular}

'able 2. Surgical outcomes in the matched population

\begin{tabular}{|c|c|c|c|}
\hline suture & Continuous (252) & Interrupted (196) & $\mathrm{p}$ values \\
\hline Estimated blood loss(EBL) (ml), Mean \pm SD & $11.4 \pm 6.8$ & $12.5 \pm 7.4$ & 0.41 \\
\hline Transfused patients, N (\%) & $53(21.0)$ & $39(19.9)$ & 0.38 \\
\hline Durations of parenteral nutrition, days, Mean \pm SD & $2.4 \pm 1.5$ & $3.1 \pm 1.2$ & 0.061 \\
\hline Early ileus, N (\%) & $59(23.4)$ & $48(24.5)$ & 0.44 \\
\hline Albumin (g/L, normal range, 35-50) & $32.9 \pm 7.6$ & $31.8 \pm 6.9$ & 0.19 \\
\hline CRP at POD 5 (mg/L, normal value: $0-8)$ & $10.6 \pm 5.8$ & $12.8 \pm 6.6$ & 0.032 \\
\hline Overall expenditure(Chinese yuan, $10^{3}$ ), Mean \pm SD & $23.8 \pm 9.4$ & $27.4 \pm 11.2$ & 0.009 \\
\hline Postoperative hospital stay, days & $8.6 \pm 1.4$ & $9.1 \pm 1.7$ & 0.09 \\
\hline $\begin{array}{l}\text { Operative time (mins), Mean } \pm \text { SD } \\
\text { anastomosis time, min, } n=10 \\
\text { Anastomosis expenditure(Chinese yuan) } n=10\end{array}$ & $\begin{array}{l}111.6 \pm 48.6 \\
11.6 \pm 3.8 \\
100\end{array}$ & $\begin{array}{l}124.1 \pm 54.2 \\
24.3 \pm 5.9 \\
21\end{array}$ & $\begin{array}{l}0.041 \\
<0.01\end{array}$ \\
\hline
\end{tabular}


Table 3. Postoperative complications in the matched population (chi-square test)

\begin{tabular}{|l|l|l|l|}
\hline \multicolumn{1}{|c|}{ suture } & Continuous (252) & Interrupted (196) & p Values \\
\hline Total complications( at least 1 complication), N (\%) & $49(19.4)$ & $42(21.4)$ & 0.34 \\
\hline Surgical wound infection, N (\%) & $18(7.1)$ & $16(8.2)$ & 0.41 \\
\hline Pneumonia, N (\%) & $12(4.8)$ & $11(5.6)$ & 0.42 \\
\hline Sepsis, N (\%) & $3(1.2)$ & $4(2.0)$ & 0.37 \\
\hline Peritonitis or abscess, N (\%) & $15(6.0)$ & $13(6.6)$ & 0.46 \\
\hline Anastomotic leakage, N (\%) & $2(0.8)$ & $3(1.2)$ & 0.38 \\
\hline Anastomotic strictures & 0 & 1 & 0.44 \\
\hline Hospital readmission, N (\%) & $21(8.3)$ & $23(11.7)$ & 0.15 \\
\hline Re-operation, N (\%) & $6(2.4)$ & $7(3.6)$ & 0.32 \\
\hline
\end{tabular}

